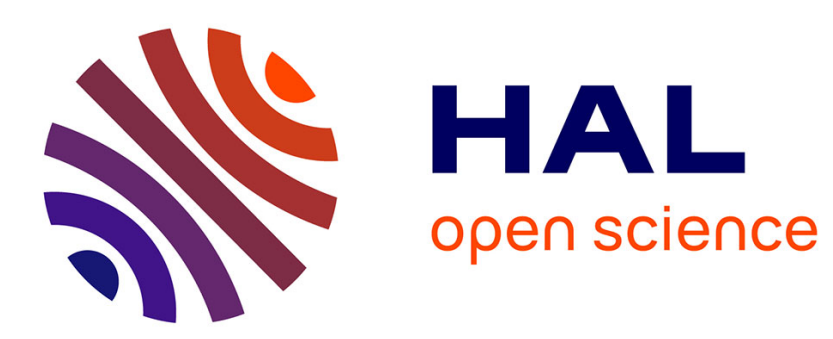

\title{
SUR LA SYNÉRESE DES CAILLÉS LACTIQUES OBTENUS PAR L'ACTION DE STREPTOCOCCUS THERMOPHILUS
}

J. Keilling, J. Casalis, Irène Glaser

\section{- To cite this version:}

J. Keilling, J. Casalis, Irène Glaser. SUR LA SYNÉRESE DES CAILLÉS LACTIQUES OBTENUS PAR L'ACTION DE STREPTOCOCCUS THERMOPHILUS. Le Lait, 1947, 27 (268), pp.449-461. hal-00927949

\section{HAL Id: hal-00927949 \\ https://hal.science/hal-00927949}

Submitted on 1 Jan 1947

HAL is a multi-disciplinary open access archive for the deposit and dissemination of scientific research documents, whether they are published or not. The documents may come from teaching and research institutions in France or abroad, or from public or private research centers.
L'archive ouverte pluridisciplinaire HAL, est destinée au dépôt et à la diffusion de documents scientifiques de niveau recherche, publiés ou non, émanant des établissements d'enseignement et de recherche français ou étrangers, des laboratoires publics ou privés. 


\title{
LE LAIT
}

REVUE GENERALE DES QUESTIONS LAITIERES

SOMMAIRE

Mémoires originaux :

J. Krilling, J. Casalts et Irène GLAsEr. - Sur la synérèse des caillés lactiques obtenus par l'action de Streptococcus thermophilus

J. Keilling, J. Casalts, Jeanne Duthem, L. Si. GONNEY et Irène GLAaER. - Sur l'accident du "bleu " en fromagerie de pâtes molles à croúte fleurie . . . .

A. Tapernoux. - Quelques problèmes d'actualité concernant l'hygiène du lait ot l'industrie laitière. . .

P. Maź. - La genèse et la persistance des éléments dégénérés dans les souches stables des ferments lactiques normaux du lait.

Bibliographie analytíque :

10 Les livres . . . . . . . 480

2. Journaux, Revues, Sociétés mavantes ........
$3 \circ$ Brevets

Bulletin bibliographique :

10 Journaux, Revues, Sociétés savantes...... 530

$2 \circ$ Brevets . . . . . . 538

Supplément technique :

G. GÉnin. - Le traitement des eaux résiduaires de laiterie

BIBLIOGRAPHIE ANALYTIQUE :

10 Revues . . . . . . . . 548

20 Brevets $\therefore . . . .552$

Documents et informations :

A. Pinel. - Etudo sur la "vache automatique ". Laiterie moderne combinée en un seul appareil . . . . 556

Communiqués ..... 5 ร8

\section{MÉMOIRES ORIGINAUX (1)}

\section{SUR LA SYNÉRÈSE DES CAILLÉS LACTIQUES OBTENUS PAR L'ACTION DE STREPTOCOCCUS THERMOPHILUS}

\author{
par
}

\section{J. KEILLING, J. CASALIS et IRÈnE GLASER}

Il arrive parfois que se produise, dans le caillage du lait par l'action des ferments lactiques thermophiles, une rétraction (synérèse) du caillé à l'intérieur des récipients qui le contiennent. Cette synérèse se manifeste par la séparation d'une phase solide (caillot)

(1) Reproduction interdite sans indioation de source, 
et d'une phase liquide (sérum). Elle nuit à la présentation commerciale de la marchandise (yoghourt et produits similaires), car les consommateurs réclament un caillé ferme, homogène, dont la surface libre soit lisse et exempte de liquide.

$$
*^{*} \text { * }
$$

Avant de préciser les conditions dans lesquelles se produit cet accident, il nous paraît opportun de rappeler rapidement la technique habituelle de fabrication de ces caillés.

La matière première utilisée actuellement est le lait écrémé. Ce dernier est soumis à un chauffage à plus ou moins haute température $\left(70\right.$ à $90^{\circ}$ C. $)$ pour réduire la flore microbienne qu'il contient. Le chauffage est parfois prolongé pendant un certain temps à l'air libre afin d'évaporer une partie de l'eau du lait et de donner au caillé une consistance plus compacte ; après chauffage, le lait est refroidi rapidement jusqu'à une température de 45 à $50^{\circ} \mathrm{C}$., puis ensemencé au moyen de ferments lactiques thermophiles et réparti dans des récipients (bouteilles ou pots) où s'effectuera la coagulation, avant la mise en vente. Ces récipients sont maintenus à l'étuve à $45^{\circ} \mathrm{C}$. jusqu'au moment du caillage. Lorsque ce dernier s'est produit, on fait subir un refroidissement énergique aux bouteilles et aux pots de façon à arrêter l'activité des ferments thermophiles.

Dans la fabrication du yoghourt, on utilise pour l'ensemencement un mélange de Streptococcus thermophitus et Thermobacterium yoghourt. Dans nos recherches, nous avons été amenés à séparer l'action de ces deux ferments et, jusqu'a présent, nous nous scmmes bornés à étudier la synérèse des eaillés obtenus au moyen de Streptococcus thermophilus.

$$
\text { ** * }
$$

La séparation du sérum et du caillé peut être attribuée à différents facteurs :

Facteurs mécaniques. Au cours des manipulations ou du transport des récipients, des trépidations accidentelles peuvent briser le caillé et a mener cette séparation.

Facteurs thermiques. Des variations brusques de température des étuves (en particulier une forte augmentation) peuvent également être la cause de l'accident. Ce dernier peut encore apparaître si l'on ne prend pas la précaution, dès le caillage, de retirer de l'étuve les récipients contenant le caillé. Dans ces conditions, les ferments continuent à se développer, l'acidification se poursuit et donne lieu à une synérèse d'autant plus marquée que le séjour à l'éture se prolonge davantage. 
Facteurs microbiens. Certaines espèces microbiennes respectées par la pasteurisation ou introduites accidentellement dans le milieu au cours des manipulations industrielles doivent être considérées comme des contaminations redoutables:

a) Soit qu'elles produisent du gaz (en général, c'est de $\mathrm{CO}^{2}$ qu'il s'agit) qui se rassemble sous forme de bulles et chemine de bas en haut en dilacérant le caillé. Cette dilacération entraîne, quelle que soit la température où elle se produit, une émission de sérum;

b) Soit qu'elles attaquent la caséine et produisent un début de digestion du coagulum.

Il est aisé de remédier à ces différentes formes de l'accident:

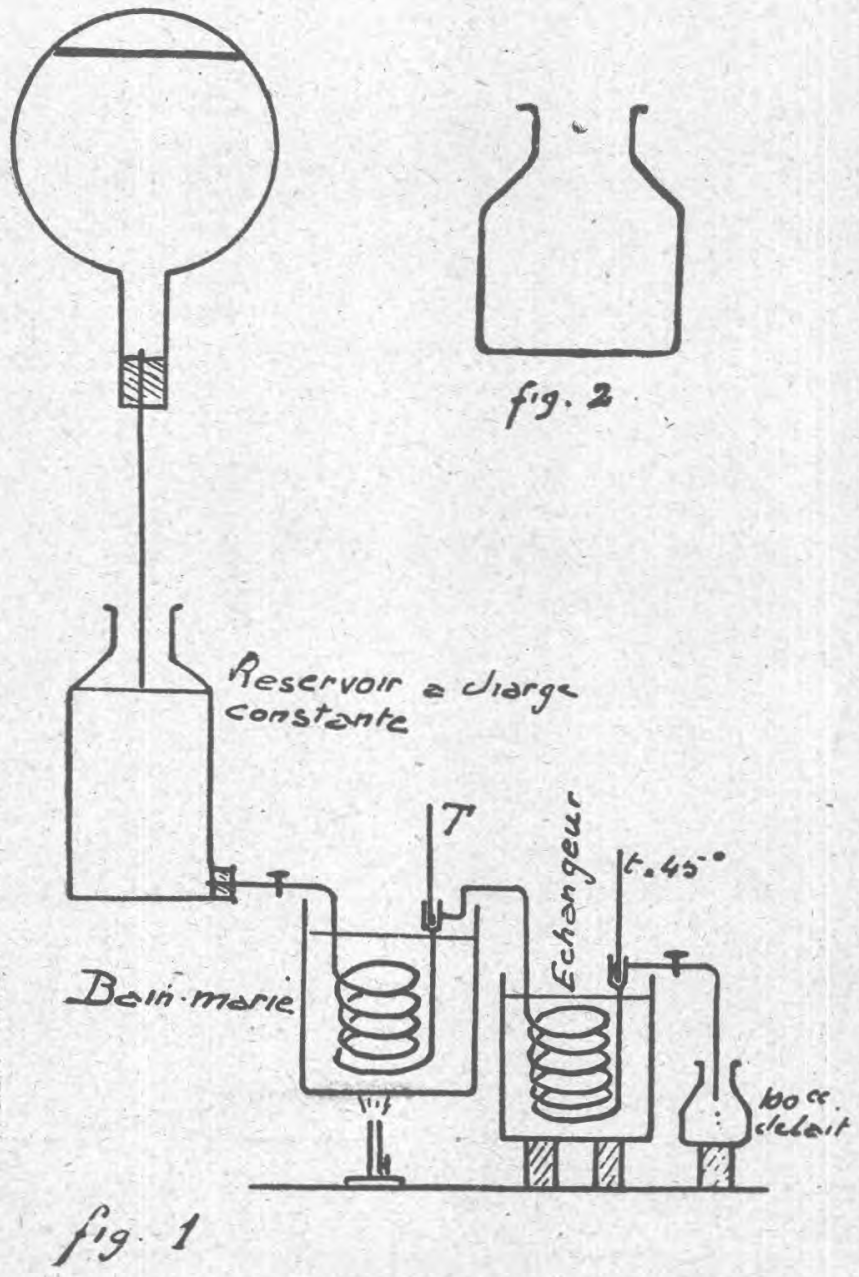


il suffit de prendre un certain nombre de précautions : éviter les: trépidations, régler et surveiller la température des étuves et le temps qu'y séjournent les récipients, la température de pasteurisation et le nettoyage du matériel.

$$
\text { * * }
$$

Mais il est une forme de l'accident dont jusqu'à présent, nous: n'avions pu expliquer le mécanisme, et qui dépend essentiellement du traitement thermique du lait avant l'ensemencement, ainsi que nous l'avons mis en évidence.

Au cours de nos expériences, un certain nombre de facteurs sont demeurés invariables:

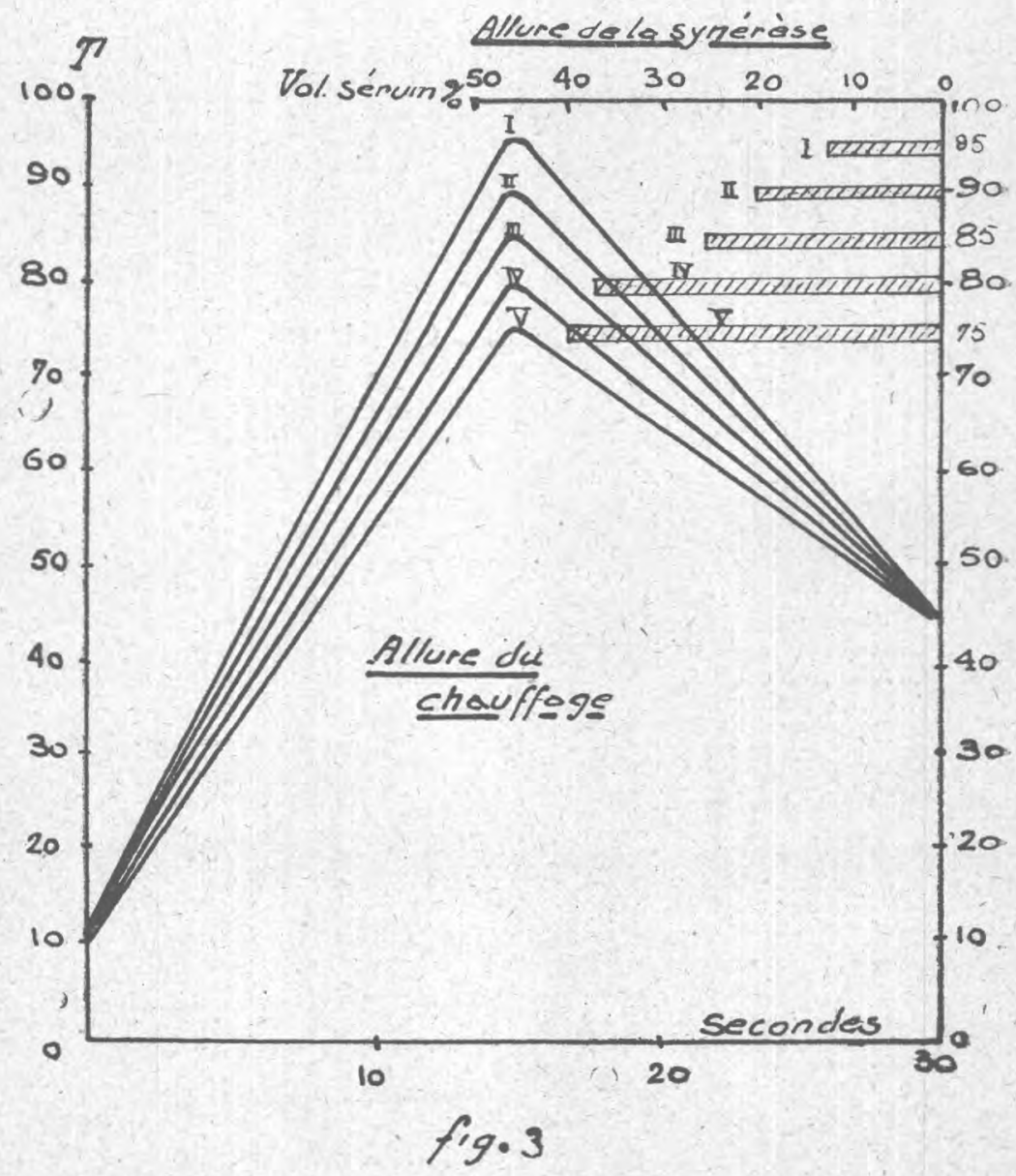




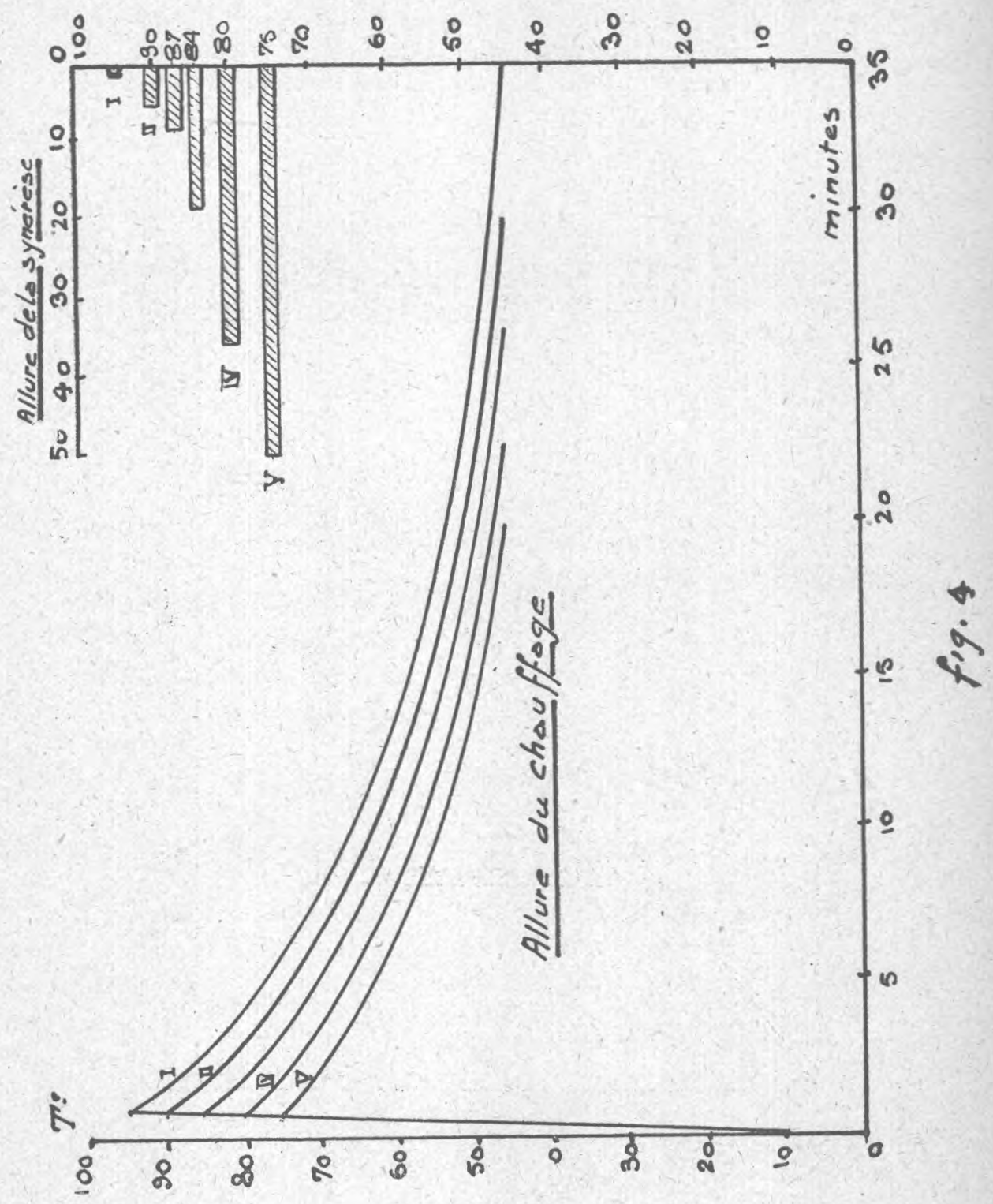

1. L'appareillage constitué par un appareil expérimental de pasteurisation que représente la figure 1.

2. Le lait utilisé pour la fabrication provenait de l'usine où se produisait l'accident ; pasteurisé avant d'être livré à l'usine, il avait, à son arrivée au laboratoire, une acidité de 18 à $20^{\circ}$ Dornic. 
3. Le levain employé à raison de $1 \%$ du volume du lait traité, et constitué par une culture pure de Streptococcus thermophilus.

4. La température d'ensemencement, $45^{\circ} \mathrm{C}$.

5. Les récipients destinés à recevoir le lait ensemencé ; vases pyrex de $125 \mathrm{~cm}^{3}$ à col large (figure 2).

6. La température de l'étuve, $45^{\circ} \mathrm{C}$.

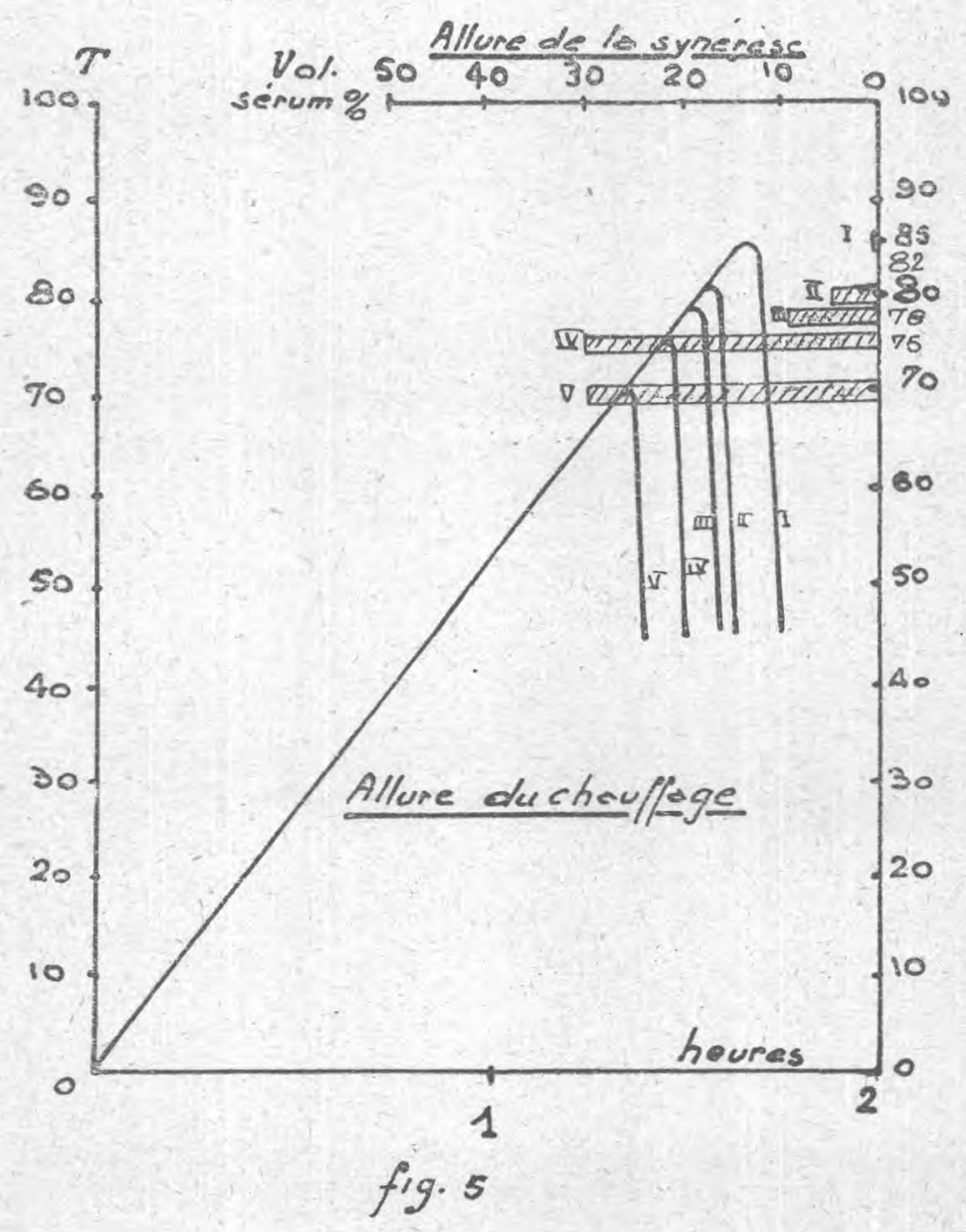

Les précautions les plus minutieuses furent prises pour éviter les contaminations microbiennes : stérilisation des appareils, nettoyage des serpentins et désinfection à l'eau javellisée.

Par contre, nous avons fait varier : la température de pasteurisation, la durée du chauffage et celle du refroidissement, au cours des expériences rapportées ci-après : 


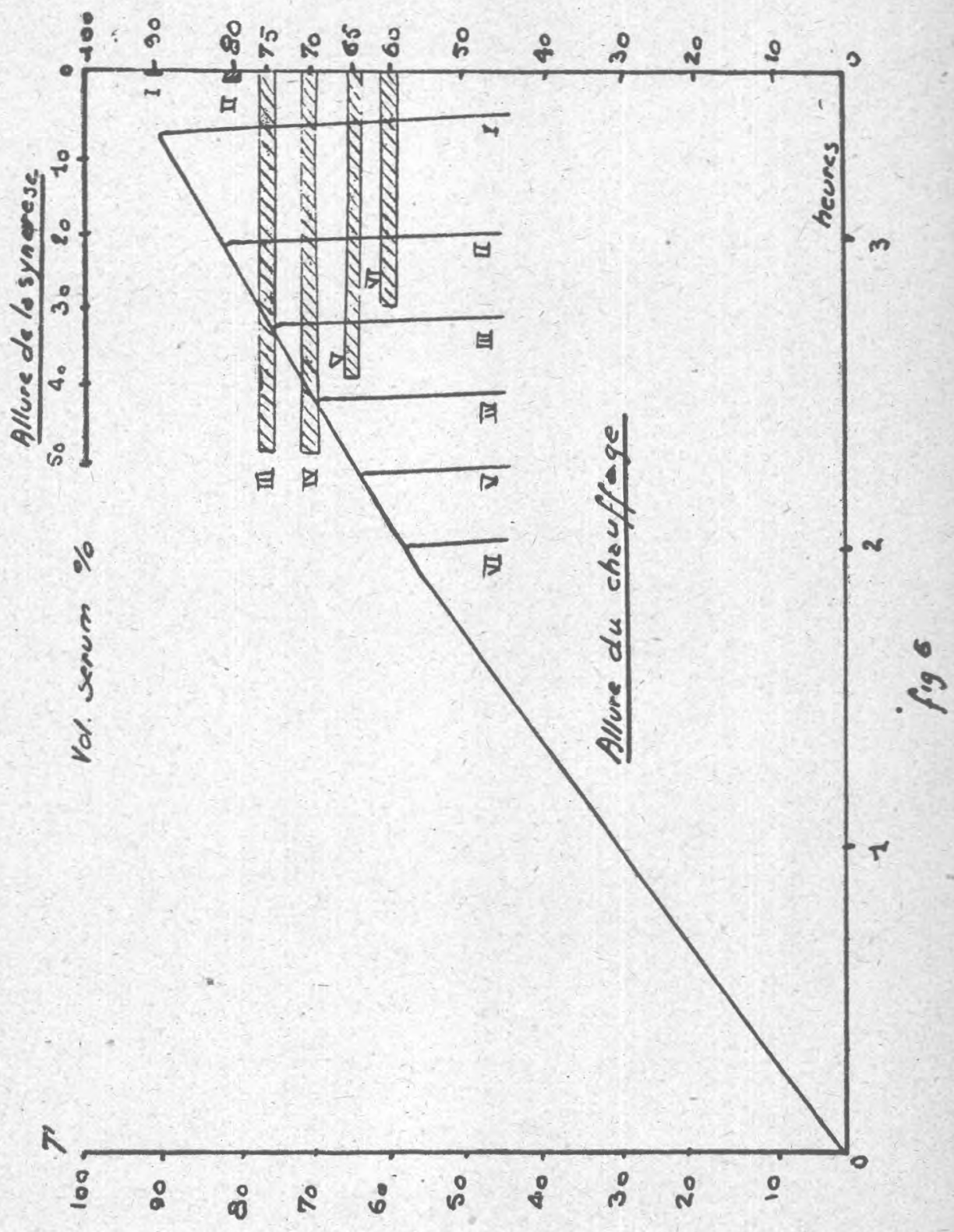

1 re expérience. Pasteurisation haute et rapide. Refroidissement rapide jusqu'à $45^{\circ} \mathrm{C}$.

Le lait est chauffé en 15 secondes, à différentes températures : $75,80,85,90,95^{\circ} \mathrm{C}$, puis refroidi en 15 secondes à $45^{\circ}$ et ensemencé (figure 3 ). 
Quelle que soit la température de ohauffage, il y a exsudation d'un sérum plus ou moins coloré et trouble. Mais le volume de ce liquide et sa coloration varient en raison inverse de la température de pasteurisation (figure 3 ). Le caillé est généralement granuleux.

$2^{\mathrm{e}}$ expérience. Pasteurisation haute et rapide. Refroidissement lent jusqu'à $45^{\circ} \mathrm{C}$.

Le lait est chauffé en 15 secondes comme dans l'expérience $n^{\circ} 1$, mais le refroidissement est spontané à l'air libre (figure 4).

On obtient des résultats semblables à ceux de la $1^{\text {re }}$ expérience (figure 4).

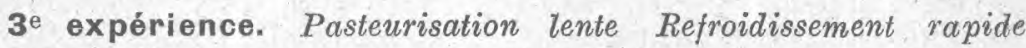
jusqu'à $45^{\circ} \mathrm{C}$.

Les figures 5 et 6 donnent la représentation graphique de deux

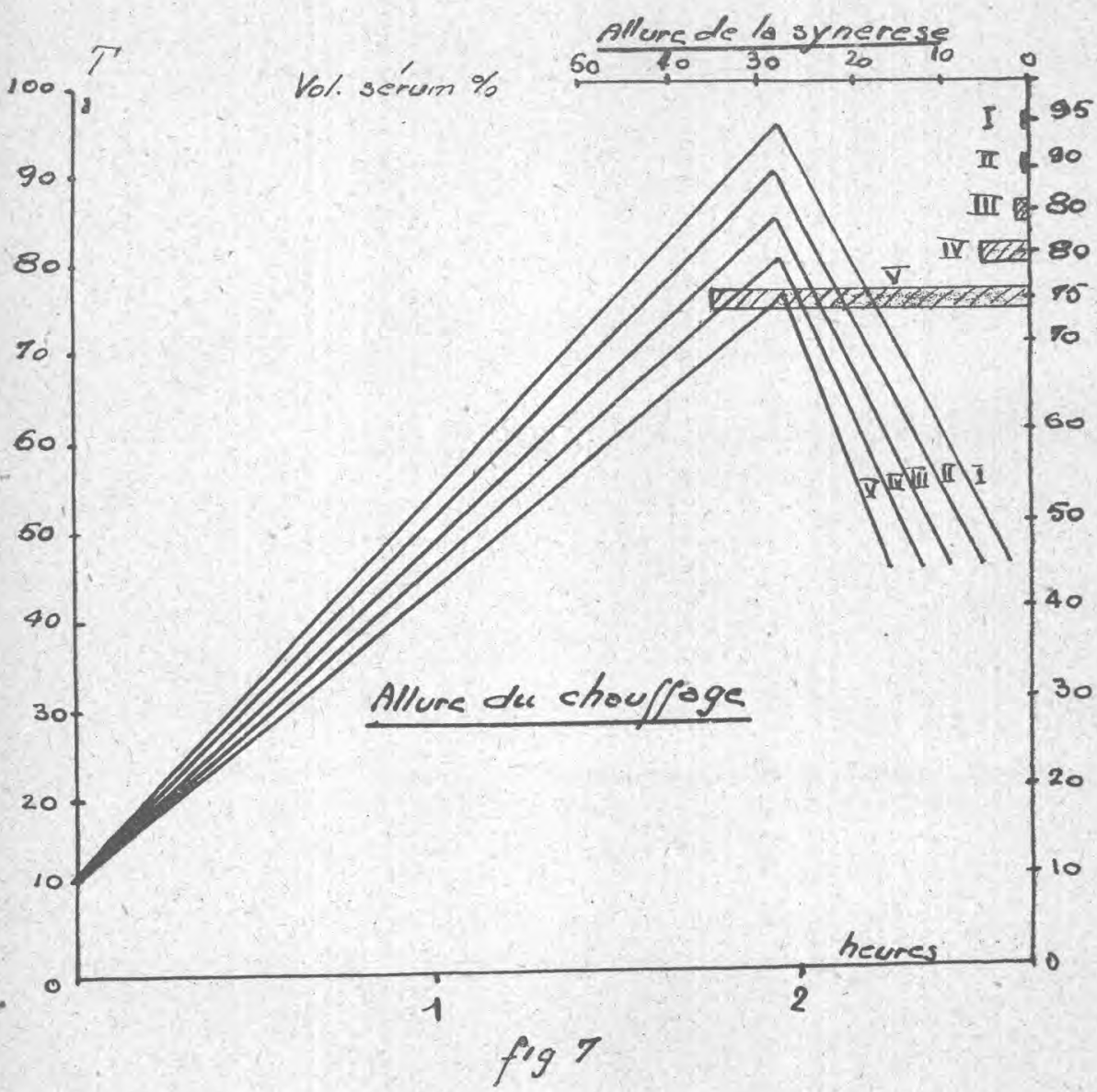


ohauffages de durée différentes. Le refroidissement est obtenu dans les deux cas en 15 secondes.

Dans l'un ou l'autre cas, l'exsudation de sérum est nulle quand la température de chauffage atteint ou dépasse $84^{\circ} \mathrm{C}$. (figure 5). Le caillé est d'autant plus ferme que cette température est plus haute. A noter toutefois qu'au-dessus de $90^{\circ} \mathrm{C}$. il se produit une très légère exsudation d'un liquide parfaitement limpide et incolore.

4 expérience. Pasteurisation lente. Refroidissement lent jusqu'à $45^{\circ} C$. (figure 7).

Les résultats sont voisins de ceux lde l'expérience 3 (figure 7). Cette expérience nous a permis de confirmer que pour des températures de pasteurisation inférieures à $82^{\circ} \mathrm{C}$., le caillé obtenu est peu consistant.

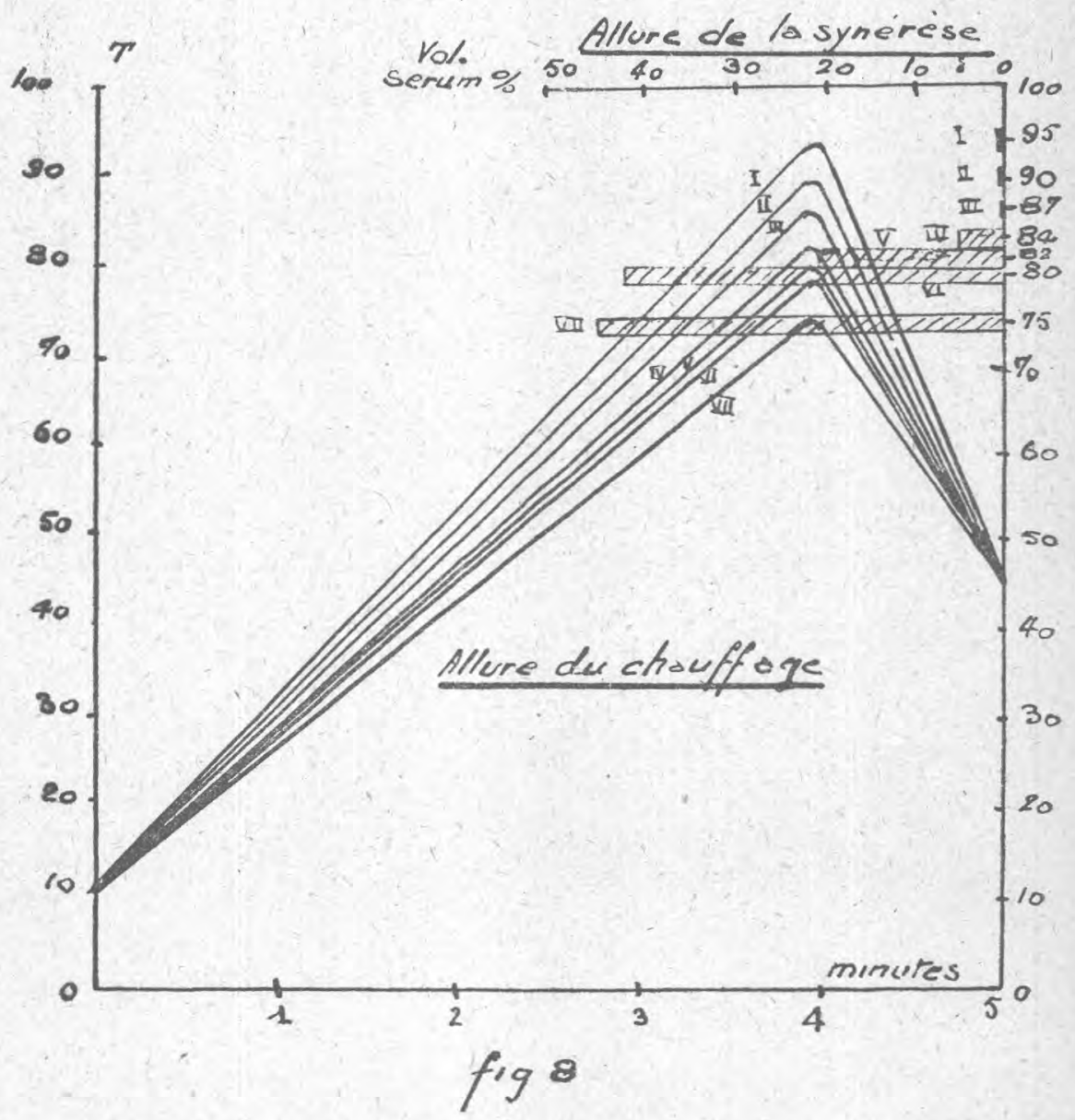


5e expérience. Cherchant à limiter les durées du chauffage, nous opérons successivement en 1 heure, 10 minutes et 4 minutes. Le refroidissement a $45^{\circ} \mathrm{C}$. est obtenu en 1 minutes Par chanffage T identiques à ceux qui sont représentés pour le chauffage $100]$ en quatre minutes (figure 8 ).

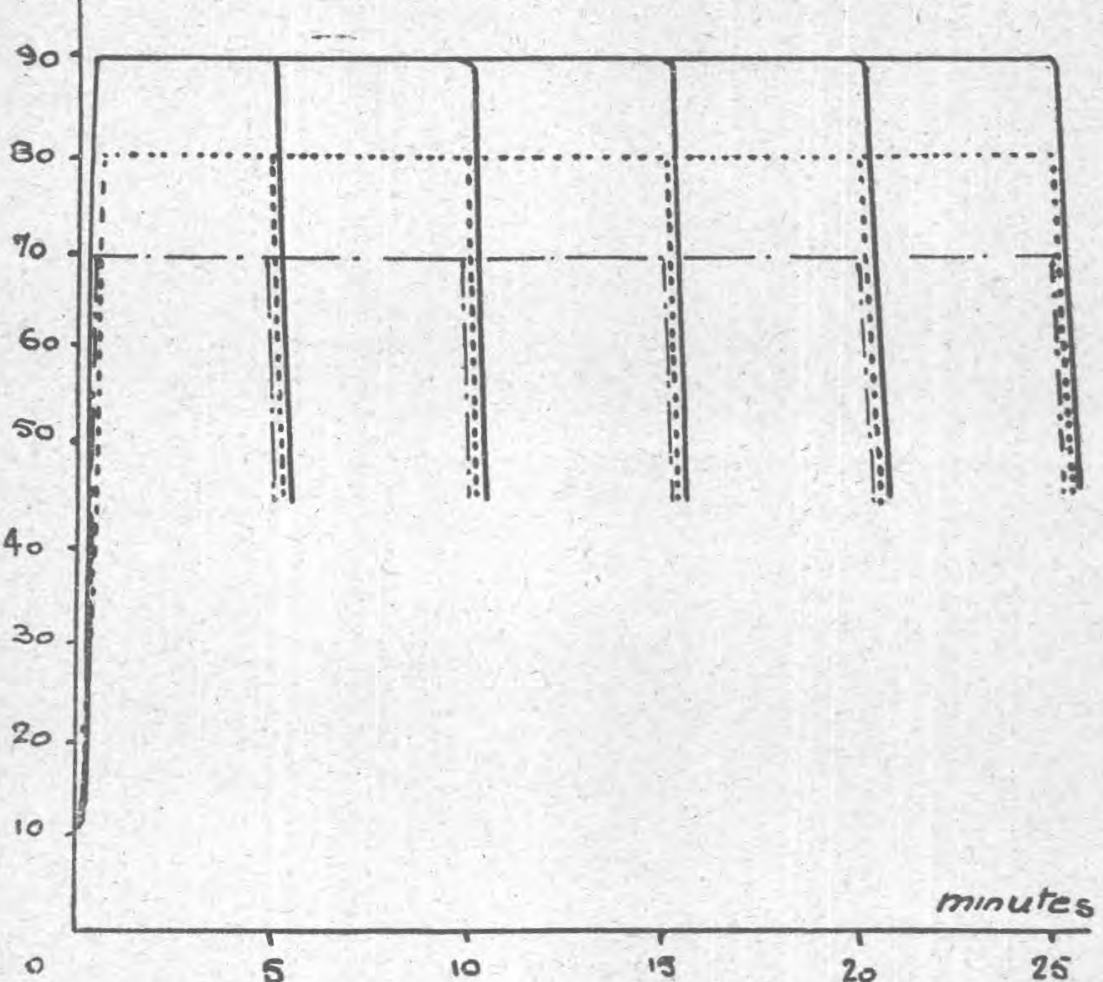

\section{fig 9}

Dans ces 3 séries d'expériences, l'exsudation de sérum est nulle quand la température de pasteurisation atteint ou dépasse $84^{\circ} \mathrm{C}$. Le caillé est d'autant plus ferme que cette température est plus haute. Au-dessus de $90^{\circ}$ il se produit une très légère exsudation d'un liquide parfaitement limpide et incolore.

6 expérience. Pasteurisation rapide suivie d'un chambrage. Refroidissement rapide jusqu'à $45^{\circ} C$. (figure 9).

Le chambrage est l'opération qui consiste à maintenir le liquide à une température donnée pendant un certain temps. 
L'exsudation de sérum se produit quelle que soit la durée du chambrage tant que la température de pasteurisation n'atteint pas $80^{\circ}$ C. A $80^{\circ} \mathrm{C}$, un chambrage de 15 minutes permet d'obtenir un caillé de consistance ferme et supprime l'exsudation de sérum. On obtient les mêmes résultats à $95^{\circ}$, avec un chambrage de 5 minutes (figure 9 bis).

En augmentant la durée du chauffage, on obtient des résultats à peu près semblables (figures 10 et 10 bis).

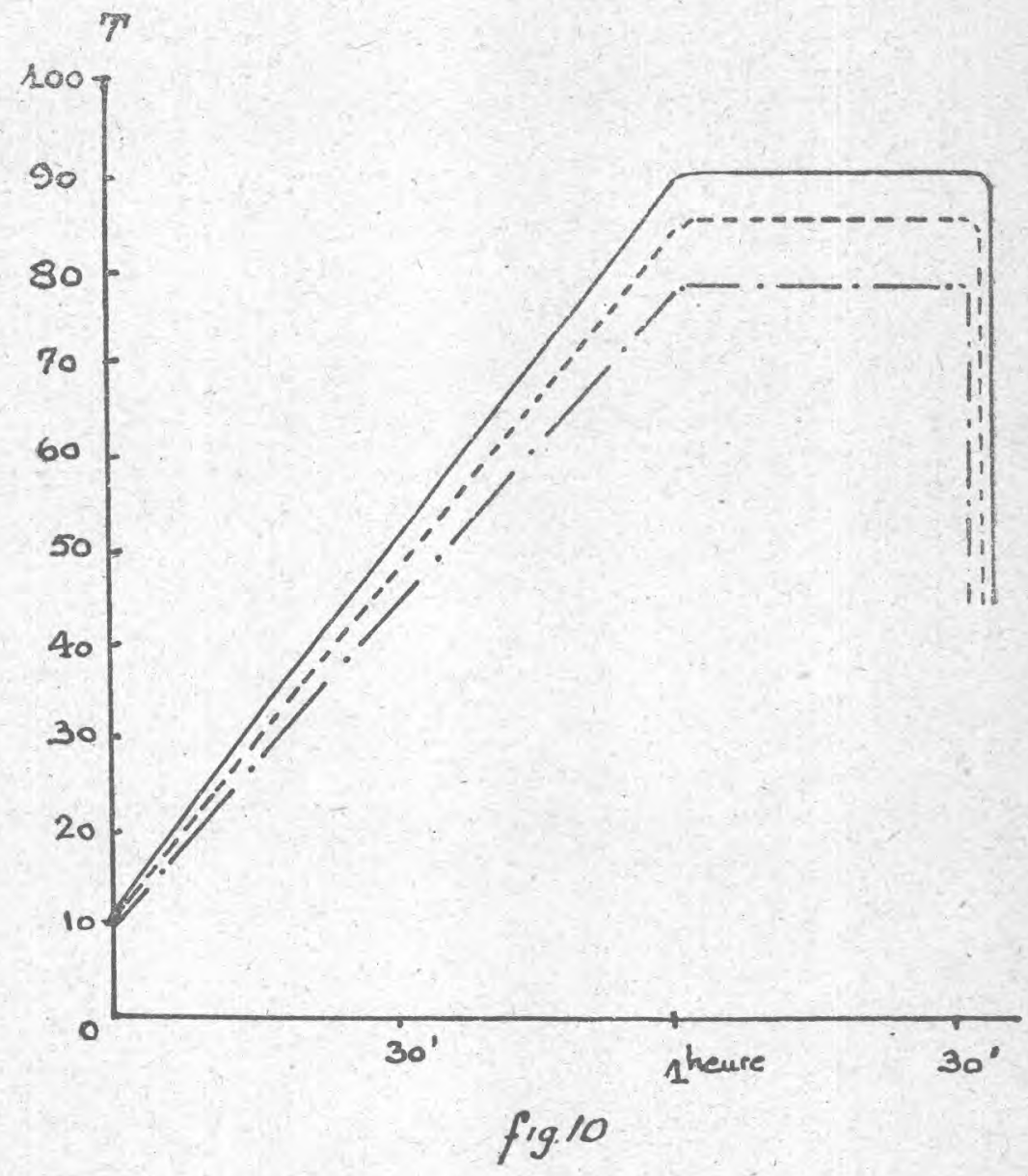

Si la durée du chauffage dépasse 25 minutes, ou si la température de pasteurisation atteint $93^{\circ} \mathrm{C}$., on obtient une légère exsudation d'un liquide incolore et limpide.

Notons, avant de conclure, que l'observation microseopique directe a permis le contrôle systématique de l'absence de conta mina- 


\section{Vol. serung \%}

50

\section{0}

30
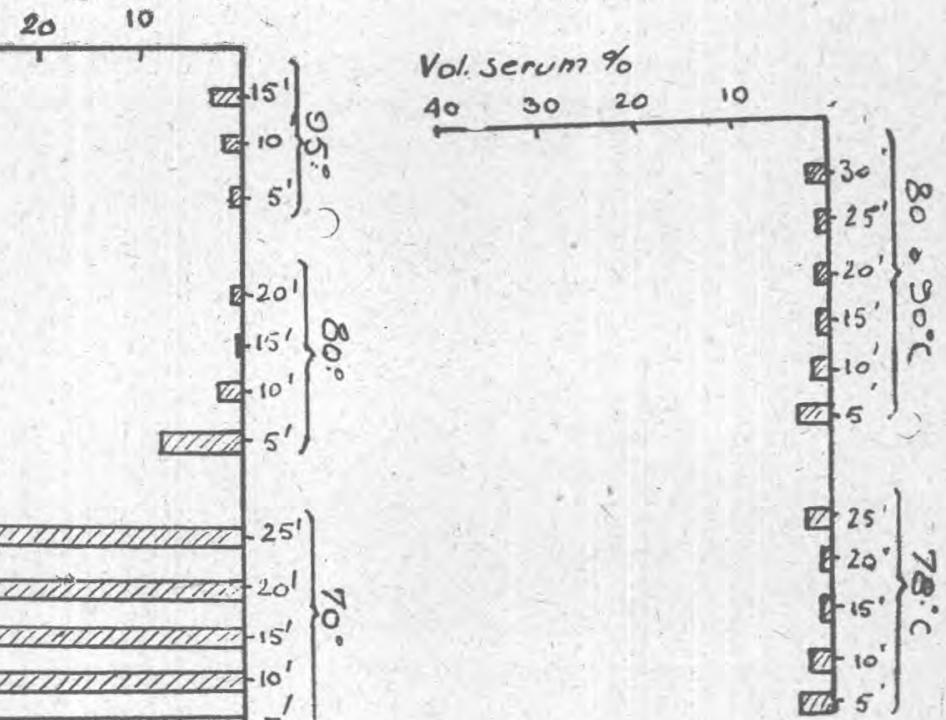

$$
\text { fig. } 96 \text { is }
$$

fig. 106 is

tions microbiennes dans les produits fabriqués au cours de ces expériences.

Chacune de celles-ci a été répétée à plusieurs reprises et les résultats obtenus ont toujours été comparables.

$$
*^{*} *
$$

Conclusions. La culture de Streptococcus thermophilus sur lait pasteurisé immédiatement avant l'ensemencement donne un caillé homogène, consistant, qui ne laisse pas exsuder de sérum dans les conditions suivantes :

1. Si le lait est porté en 15 secondes à $80^{\circ} \mathrm{C}$. et maintenu $15 \mathrm{mi}-$ nutes à cette température.

Si le lait est porté en 15 secondes à $90^{\circ} \mathrm{C}$. et maintenu 5 minutes à cette température.

2. Si le lait est porté en 4 minutes à $84^{\circ} \mathrm{C}$., sans qu'il soit besoin, dans ce cas, de faire suivre le chauffage d'un chambrage.

Des études complémentaires nous permettront de raccorder le phénomène que nous venons de signaler aux problèmes similaires, 
d'ordre physico-chimique, que pose la fromagerie qui travaille des laits pasteurisés.

(Travail de la Station centrale de Technologie agricole de l'Institut national de la Recherche agronomique.)

\title{
SUR L'ACCIDENT DU «BLEU » EN FROMAGERIE DE PATES MOLLES A CROUTE FLEURIE
}

\author{
par \\ J: KEILLING, J. CASALIS, JEANNE DUTHEN, L. SIGONNEY \\ et Irène GLASER
}

Parmi les difficultés que rencontrent les fromagers de pâtes molles à croûte "fleurie» (Camembert, Coulommiers, Brie, Carré de l'Est); la maladie du “Bleu " se place au premier rang. Elle se caractérise par la présence à la surface du fromage, au milieu de la couche blanche de Penicillium candidum, de taches bleuâtres ou verdatres de plus ou moins grandes dimensions. Le "Bleu " apparait sous forme soit de colonies bien localisées, soit, au contraire, de taches s'étendant sur tout ou partie de l'une ou l'autre des faces des fromages.

Cette altération qui n'affecte pas, le plus souvent, la saveur des produits, mais toujours leur présentation commerciale, est due à une modification de la flore fongique normale des fromages : Penicillium glaucum y apparaît de façon plus ou moins marquée. Le mycélium de ce champignon est blanc, mais ses spores sont fortement colorées ; l'apparition des spores et, par voie de conséquence, de la coloration, peut se produire très brusquement au milieu du feutrage blanc de Penicillium candidum, et cette rapidité d'évolution explique l'étonnement des praticiens qui voient la couleur blanche des fromages passer au bleu en l'espace de quelques heures.

Depuis toujours on a préconisé et pratiqué, pour la lutte contre cet accident, à la fois la désinfection totale des locaux et le contrôle de la pureté des cultures de Penicillium candidum utilisées pour l'ensemencement des fabrications. Penicillium glaucum est une moisissure très abondante dans la nature, et il trouve dans les locaux ou le matériel des usines laitières un milieu extrêmement favorable à son développement. D'autre part, les suspensions ou les poudres de spores de Penicillium candidum que le commerce met à la disposition des fabricants pour I'ensemencement des fromages peuvent accidentellement être contaminées au cours de leur fabrication par des spores de Penicillium glaucum.

Le plus généralement, t:ne désinfection systématique des locaux, accompagnée de l'ensemencement au moyen de suspensions de 